University of Windsor

Scholarship at UWindsor

$12-31-2019$

\title{
Photophysical and Optical Properties of Semiconducting Polymer Nanoparticles Prepared from Hyaluronic Acid and Polysorbate 80
}

\author{
Adam Langlois \\ University of Windsor \\ Gage T. Mason \\ University of Windsor \\ Michael H.L. Nguyen \\ University of Windsor \\ Mehdi Rezapour \\ University of Windsor \\ Paul Ludovic Karsenti \\ Université de Sherbrooke
}

See next page for additional authors

Follow this and additional works at: https://scholar.uwindsor.ca/chemistrybiochemistrypub

Part of the Biochemistry, Biophysics, and Structural Biology Commons, and the Chemistry Commons

\section{Recommended Citation}

Langlois, Adam; Mason, Gage T.; Nguyen, Michael H.L.; Rezapour, Mehdi; Karsenti, Paul Ludovic; Marquardt, Drew; and Rondeau-Gagné, Simon. (2019). Photophysical and Optical Properties of Semiconducting Polymer Nanoparticles Prepared from Hyaluronic Acid and Polysorbate 80. ACS Omega, 4 (27), 22591-22600.

https://scholar.uwindsor.ca/chemistrybiochemistrypub/153

This Article is brought to you for free and open access by the Department of Chemistry and Biochemistry at Scholarship at UWindsor. It has been accepted for inclusion in Chemistry and Biochemistry Publications by an authorized administrator of Scholarship at UWindsor. For more information, please contact scholarship@uwindsor.ca. 


\section{Authors}

Adam Langlois, Gage T. Mason, Michael H.L. Nguyen, Mehdi Rezapour, Paul Ludovic Karsenti, Drew Marquardt, and Simon Rondeau-Gagné 


\title{
Photophysical and Optical Properties of Semiconducting Polymer Nanoparticles Prepared from Hyaluronic Acid and Polysorbate $\mathbf{8 0}$
}

\author{
Adam Langlois, ${ }^{\dagger} \| \odot$ Gage T. Mason, ${ }^{\dagger, \|}$ Michael H. L. Nguyen, ${ }^{\dagger}$ Mehdi Rezapour, $^{\dagger}$

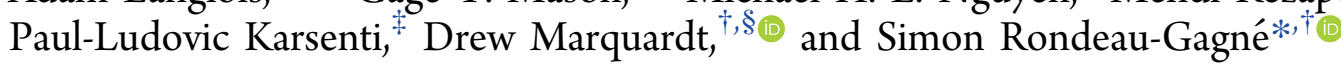

${ }^{\dagger}$ Department of Chemistry and Biochemistry, Advanced Materials Centre of Research (AMCORe) and ${ }^{\S}$ Department of Physics, University of Windsor, 401 Sunset Avenue, Windsor, Ontario N9B 3P4, Canada

${ }^{\ddagger}$ Département de Chimie, Université de Sherbrooke, Sherbrooke, Québec J1K 2R1, Canada

\section{Supporting Information}

ABSTRACT: A nanoprecipitation procedure was utilized to prepare novel diketopyrrolopyrrole-based semiconducting polymer nanoparticles (SPNs) with hyaluronic acid (HA) and polysorbate 80 . The nanoprecipitation led to the formation of spherical nanoparticles with average diameters ranging from 100 to $200 \mathrm{~nm}$, and a careful control over the structure of the parent conjugated polymers was performed to probe the influence of $\pi$-conjugation on the final photophysical and thermal stability of the resulting SPNs. Upon generation of a series of novel SPNs, the optical and photophysical properties of the new nanomaterials were probed in solution using various techniques including transmission electron microscopy, dynamic light scattering, small-angle neutron scattering, transient absorption, and UV-vis spectroscopy. A careful comparison was performed between the different SPNs to evaluate their

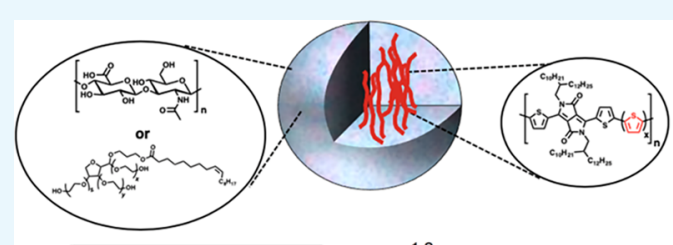
excited-state dynamics and photophysical properties, both before and after nanoprecipitation. Interestingly, although soluble in organic solution, the nanoparticles were found to exhibit aggregative behavior, resulting in SPNs that exhibit excited-state behaviors that are very similar to aggregated polymer solutions. Based on these findings, the formation of HA- and polysorbate 80-based nanoparticles does not influence the photophysical properties of the conjugated polymers, thus opening new opportunities for the design of bioimaging agents and nanomaterials for healthrelated applications.

\section{INTRODUCTION}

Organic semiconducting polymers are a special class of materials that have been attracting a lot of attention for a rich variety of applications in optoelectronics, mostly because of their intrinsic charge transport properties, exceptional optical properties, and synthetic versatility. ${ }^{1-3}$ Throughout the years, $\pi$-conjugated polymers have been used as active materials in high-performance electronic devices such as organic field-effect transistors, ${ }^{4,5}$ organic photovoltaics, ${ }^{6,7}$ and organic light-emitting diodes. ${ }^{8,9}$ These materials have also been shown to be particularly promising candidates for the development of advanced stretchable and conformable bioelectronics and sensing devices. ${ }^{10-13}$ Recently, it has been shown that by employing different preparation strategies, $\pi$ conjugated polymers can be aggregated in solution and stabilized by hydrophilic polymers, leading to the preparation of a new type of nanomaterials, the so-called semiconducting polymer nanoparticles (SPNs). ${ }^{14-16}$ Interestingly, SPNs are easily accessible synthetically, present good stability in aqueous dispersion, and their size can be easily fine-tuned. More importantly, SPNs were shown to preserve the rich optical properties of the native semiconducting polymer, which led to their evaluation as bioimaging agents (near infrared, NIR) and potential application in the treatment of various diseases through photothermal therapy. ${ }^{17-19}$ Currently, most approaches for labeling and visualizing extracellular materials (proteins, polysaccharides, lipids, etc.) use commercial fluorescent compounds, such as fluorescein isothiocyanate and rhodamine. ${ }^{20}$ Despite being well known and accessible, these compounds can suffer from leaching and poor photostability, which remain important for long-term tracking and imaging purposes. Therefore, the design of novel SPNs and the investigation of their optical and photophysical properties are highly desirable toward a more efficient platform for bioimaging and therapeutics.

For the preparation of novel SPNs through nanoprecipitation, the utilization of dye molecules capable of absorbing in the NIR range has been shown to be a promising design strategy to access improved optical properties. ${ }^{21,22}$ Among others, diketopyrrolopyrrole (DPP) is a good candidate for designing new SPNs for various reasons. ${ }^{4,23,24}$ First, DPP is a planar electron-deficient unit that is synthetically tunable, and

Received: October 13, 2019

Accepted: December 5, 2019

Published: December 19, 2019 


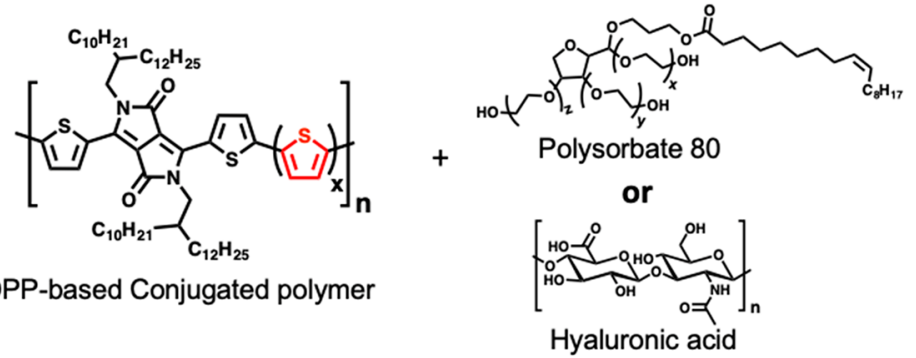

Nanoprecipitation

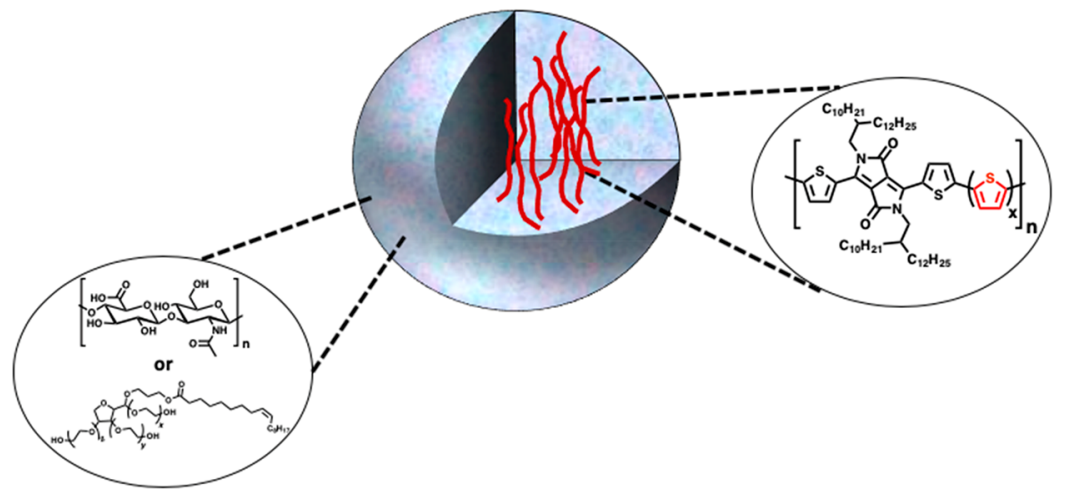

Figure 1. Formation of DPP-based conjugated polymer nanoparticles (SPNs) with HA or polysorbate 80 (Tween 80) through nanoprecipitation.

its preparation is well known because of the fact that it has been widely used for optoelectronic applications. Moreover, DPP-based materials typically possess excellent light and thermal stability, making them particularly suitable for laser cellular tracking through photoacoustic imaging. Finally, the energy gaps can be easily tuned through copolymerization with other monomers, thus enabling a fine-tuning of its absorption and photophysical properties. Many recent SPN systems have been reported using DPP-based materials and were shown to be particularly efficient for photothermal stability and photoacoustic imaging. ${ }^{25-27}$ Despite these important outcomes, the investigation of the synthetic design rules to fully understand the fine-tuning of the photophysical properties of DPP-based SPNs and the utilization of new hydrophilic coprecipitation agents with novel properties are still highly desirable.

Herein, we report the preparation of new SPNs through the nanoprecipitation of a series of DPP-based conjugated polymers with hyaluronic acid (HA) and polysorbate 80 (Figure 1). Widely used for the design of novel biomaterials, $\mathrm{HA}$ and polysorbate 80 are known to be specific targets of specific receptors of various tumors, mostly associated with glioblastoma. ${ }^{28-32}$ Furthermore, both biomaterials have been previously utilized transitioning nanoparticles across the blood-brain barrier, which is particularly interesting for the design of new SPNs. ${ }^{33,34}$ In addition to the utilization of new hydrophilic coprecipitation agents, a systematic evaluation was performed by varying the main chain structure by inserting different donor moieties, including thiophene $(\mathrm{T})$, bithiophene (2T), and terthiophene (3T) into the DPP polymer to probe for the impact of $\pi$-conjugation extension on the photophysical properties of SPNs before and after nanoprecipitation. The new SPNs were characterized by various techniques, including UV-vis spectroscopy, dynamic light scattering (DLS), small- angle neutron scattering (SANS), fluorescence spectroscopy, and femtosecond transient absorption (TA) spectroscopy measurements, to unveil their photophysical properties and the effect of the molecular design of the nanoparticles. The indepth characterization of the new SPNs revealed that SPNs prepared from HA and polysorbate 80 exhibit fast kinetic decays of the excitons upon irradiation as measured by TA spectroscopy. Interestingly, the incorporation of various thiophene moieties or the utilization of HA of Tween 80 does not significantly impact the photophysical properties, and the newly prepared nanoparticles maintained the optical properties of their parent polymers (before nanoprecipitation). Ultimately, the investigation and evaluation of the new HA and polysorbate DPP-based SPNs can potentially enable the finetuning of the optical properties of such materials, contributing to the preparation new high-performance imaging and therapeutic platforms.

\section{RESULTS AND DISCUSSION}

The three different DPP-based conjugated polymers (Figure 2a), namely, P(DPP-T), P(DPP-2T), and P(DPP-3T), were prepared according to previous reports. ${ }^{38}$ Briefly, a decyltetradecyl-branched diketopyrrolopyrrole monomer was copolymerized with different stannylated derivatives via Stille polymerization. The resulting polymer was precipitated with methanol, purified by Soxhlet extraction with methanol, acetone, and hexane, collected with chloroform before being precipitated in methanol, and dried under vacuum. As shown in Figures S1-S9, nuclear magnetic resonance (NMR) and Fourier transform infrared spectroscopies were used to confirm the structure of the conjugated polymers. Upon synthesis, the DPP-based polymers were characterized by high-temperature gel permeation chromatography (GPC) to probe for their 
a)

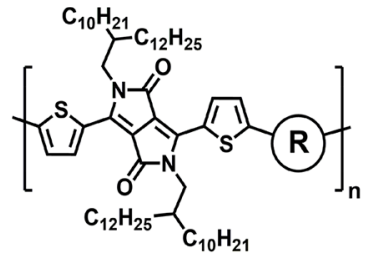

b)

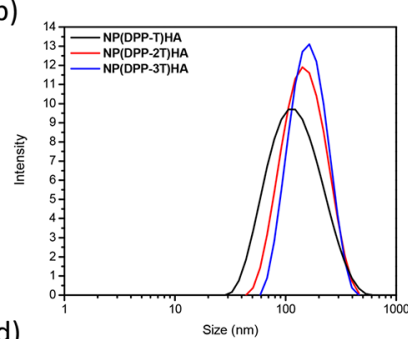

d)

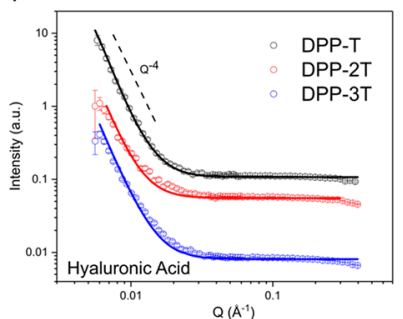

$\mathrm{P}(\mathrm{DPP}-\mathrm{T}), \mathrm{R}=\gamma^{\mathrm{S}} \mathrm{I}$

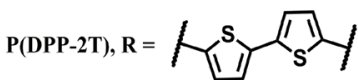

$\mathrm{P}(\mathrm{DPP}-3 \mathrm{~T}), \mathrm{R}=\mathrm{H}_{\mathrm{S}}^{\mathrm{s}}$

c)

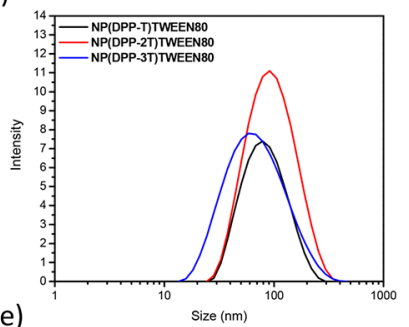

e)

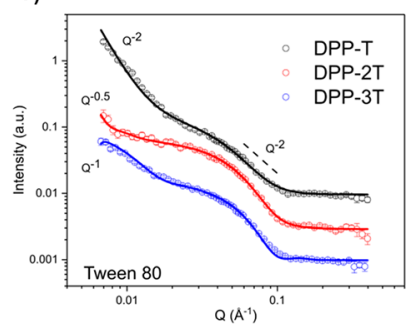

Figure 2. (a) Structures of the investigated semiconducting polymers P(DPP-T), P(DPP-2T), and P(DPP-3T); size distributions of SPN samples prepared from (b) HA and (c) Tween-80 as measured by DLS. SANS data of SPNs in $\mathrm{D}_{2} \mathrm{O}$ prepared from (d) HA and (e) Tween-80. Solid lines represent fits. Dashed lines show Q-dependence of the sample scattering. $I(Q)$ scattering curves were vertically offset using scaling factors for clarity of presentation.

molecular weight. As shown in Table S1, P(DPP-T), P(DPP$2 \mathrm{~T})$, and P(DPP-3T) showed a molecular weight of $13.7,22.2$, and $20.6 \mathrm{kDa}$, respectively, with polydispersity indexes around 2. It is important to mention that Carothers' equation was used during polymerization in order to limit the molecular weights, thus allowing for a better control over the solubility of the polymers in the desired solvents used for nanoprecipitation. Optical properties, energy levels, and decomposition temperatures of $\mathbf{P}(\mathbf{D P P}-\mathrm{T}), \mathbf{P}(\mathbf{D P P}-2 \mathrm{~T})$, and $\mathbf{P}(\mathbf{D P P}-3 \mathrm{~T})$ are also depicted in Table $\mathrm{S} 1$.

Following the synthesis of the semiconducting polymers, preparation of the SPNs was undertaken using commercially available hydrophilic polymers HA and polysorbate 80 (Tween 80 ). The complete procedure for nanoprecipitation is detailed in the Supporting Information. Upon addition of the various precursors to water under sonication, rapid diffusion of the solvent and aqueous phase leads to supersaturation of the polymer, thus forming nanoparticles which are stabilized by the

hydrophilic materials. Upon formation of the SPNs, namely, NP(DPP-T), NP(DPP-T2), and NP(DPP-T3), the samples were purified through successive washes with water and filtered using a $0.2 \mu \mathrm{m}$ filter to remove any large undispersed polymer aggregates. Importantly, all nanoparticle suspensions in water shown good stability, up to a month at $4{ }^{\circ} \mathrm{C}$. However, as shown in Figure S10, the samples began to decompose after 2 weeks at ambient conditions, confirmed by the appearance of smaller features observed by DLS.

The SPNs were first analyzed by DLS to probe for size distributions and dispersity, and the results are shown in Table 1 and Figure 2b,c. Interestingly, independently of the semiconducting core, the SPNs showed relatively narrow size distribution with an average size of $133 \mathrm{~nm}$ for NP(DPP-T), $150 \mathrm{~nm}$ for NP(DPP-2T), and $171 \mathrm{~nm}$ for NP(DPP-3T) when HA was used as a stabilizing hydrophilic polymer. Similarly, an average size of $89 \mathrm{~nm}$ for NP(DPP-T), $104 \mathrm{~nm}$ for NP(DPP-2T), and $79 \mathrm{~nm}$ for NP(DPP-3T) were observed when Tween 80 was used. These results show the relatively similar behavior of the polymer in aqueous media, in terms of aggregation and stability. To confirm their size and investigate the shape and morphology, SPNs were subjected to SANS. All SPNs optimally modeled using a spherical uniform scattering length density, with a sphere radius $\left(R_{\mathrm{s}}\right)$ which correlates with the hydrodynamic radius $\left(R_{\mathrm{h}}\right)$ determined from DLS (Table $1)$. The spherical shape and sizes of SPNs are corroborated by transmission electron microscopy (TEM), and the results are summarized in Figure S11. The SANS data further yielded the radius of gyration $\left(R_{\mathrm{g}}\right)$ of the SPNs, which appears invariable to the number of thiophenes present in the polymer. Insight into the geometry of the particles can be inferred from the $R_{\mathrm{g}} /$ $R_{\mathrm{h}}$ ratio, where $R_{\mathrm{g}} / R_{\mathrm{h}}=0.775$ for hard spheres. ${ }^{39,40}$ The SPNs presented here yield $R_{\mathrm{g}} / R_{\mathrm{h}}$ values $0.63-0.73$ (polysorbate 80 ) and $0.41-0.53(\mathrm{HA})$. These results support a spherical geometry, as $R_{\mathrm{g}} / R_{\mathrm{h}}$ values $>0.775$ are a result of rodlike and disklike geometries, and values $<0.775$ have been associated with spherical particles that possess long-range interactions and aggregation in solution. ${ }^{40,41}$ Q-dependence analysis of the SANS data shows that the number of thiophenes and surfactants used can influence the SPN structure and shape (Figures 2d,e and S12). SPNs formed with HA consistently follows a power law of $\sim Q^{-4}$, which typically describes a compact, spherical particle with sharp interfaces. For SPNs with Tween 80 , changes in scattering intensity in the low $Q$ region occur depending on thiophene units. This may be due to a difference in aggregative behavior or may occur when SPN micellar shape shifts from rodlike $\left(Q^{-1}\right)$ to lamellar or disklike $\left(Q^{-2}\right)$, and vice versa. The mid $Q$-dependence of $Q^{-2}$ found in all three SPNs with Tween 80 is also indicative of markedly flexible micelles. ${ }^{42}$

Table 1. Average Diameter and Dispersity for SPNs Prepared from Polysorbate 80 and HA as Measured by DLS ${ }^{a}$

$\begin{array}{clccccc}\text { hydrophilic polymer } & \text { polymer } & \text { average diameter, } 2 R_{\mathrm{h}}(\mathrm{nm}) & \text { PDI } & R_{\mathrm{g}}(\mathrm{nm}) & R_{\mathrm{g}} / R_{\mathrm{h}}^{*} & R_{\mathrm{s}}(\mathrm{nm}) \\ \text { polysorbate } 80 & \text { NP(DPP-T) } & 89 & 0.41 & 28 & 0.63 & 43.4 \\ & \text { NP(DPP-2T) } & 104 & 0.26 & 33 & 0.63 & 53.1 \\ & \text { NP(DPP-3T) } & 79 & 0.46 & 29 & 0.73 & 17.1 \\ \text { HA } & \text { NP(DPP-T) } & 133 & 0.52 & 35.4 & 0.53 & 36.9 \\ & \text { NP(DPP-2T) } & 150 & 0.28 & 35.2 & 0.47 & 43.4 \\ & \text { NP(DPP-3T) } & 171 & 0.25 & 34.9 & 0.41 & 55.6\end{array}$

${ }^{a}$ Radius of gyration $\left(R_{\mathrm{g}}\right)$ and radius of the SPN $\left(R_{\mathrm{s}}\right)$ as measured by SANS. 
a)

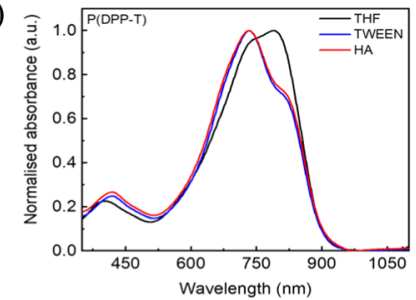

b)

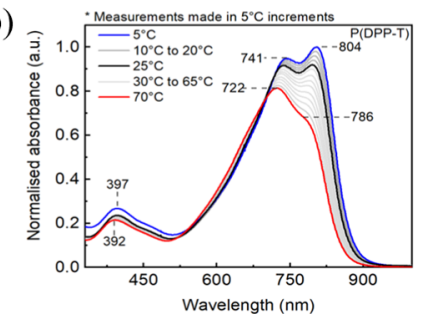

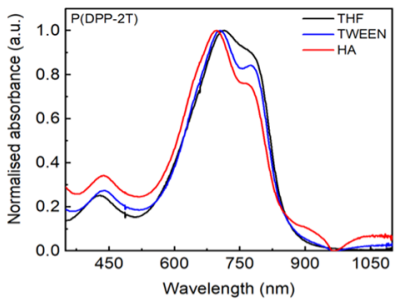

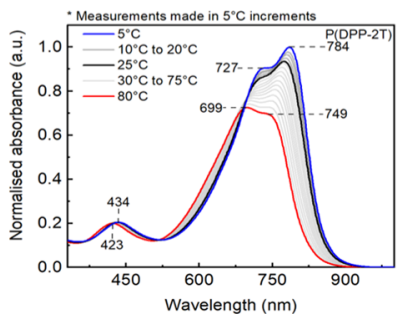

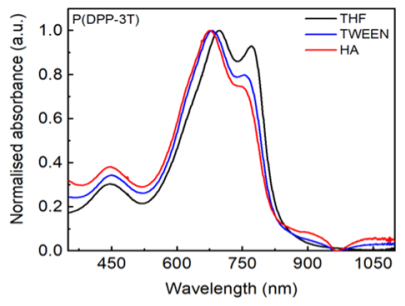

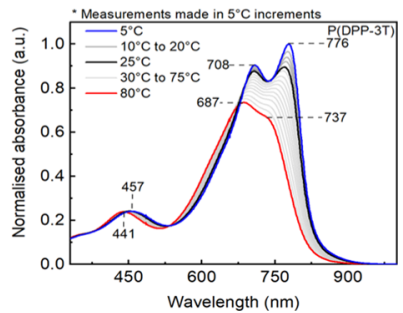

Figure 3. (a) UV-vis absorption spectra of DPP-based polymers in tetrahydrofuran (THF) solution before and after nanoprecipitation with HA and Tween 80 and (b) variable temperature UV-vis (VT-UV-vis) spectra of the conjugated polymers in chlorobenzene. Spectra are obtained by slowly cooling solutions from their maximum temperature in a controlled manner.

Based on the results from SANS and DLS, all SPN samples, independent of the stabilizing hydrophilic polymer, were shown to be composed of spherical nanoparticles averaging $100-200 \mathrm{~nm}$ in diameter. Interestingly, for the SPNs prepared from Tween 80, the addition of thiophene moieties in the $\pi$ conjugated backbone led to a smaller size distribution in the resulting NPs. This can be potentially explained by an increased aggregation for $\mathbf{P}(\mathbf{D P P}-\mathbf{T})$ in solution, which is supported by a low $R_{\mathrm{g}} / R_{\mathrm{h}}$ ratio. For the NPs prepared from $\mathrm{HA}$, the particles were observed to generate more bundles than from the samples prepared from Tween 80. Despite slight difference in sizes depending on the structure of the polymer, all particles were shown to be around the same diameters and shapes. It is important to mention that nanoprecipitation was also attempted using a 1:4 hydrophilic polymer/conjugated polymer ratio, and the size distribution was investigated by DLS to probe for the influence of surfactant/semiconducting polymer ratios on the final nanoparticles. Interestingly, the NPs prepared with a $1: 4$ ratio resulted in very similar average size distributions than the NPs prepared with a 1:2 ratio, as shown in Figure S13.

Density functional theory (DFT) calculations by Gaussian 09 were also performed on P(DPP-T), P(DPP-2T), and $\mathbf{P}(\mathbf{D P P}-3 \mathrm{~T})$, and the results are summarized in Figure S14. This analysis allowed for gaining insights into the backbone conformation upon extension of the $\pi$-conjugation induced by the addition of thiophene units. After geometry optimization, a two-coordinate potential energy scan of dihedral angles was performed in order to do a conformational search on the most stable configuration. Based on the resulting potential energy scans, the most stable conformation for all three polymers is when the polymer backbone is completely planar, which has been observed for similar $\pi$-conjugated polymer systems. ${ }^{43}$ This result suggests that the addition of thiophene units has a moderate conformational influence on the $\pi$-conjugated backbone.

Evaluation of the optical properties of the DPP-based polymers before and after formation of nanoparticles was performed by UV-vis spectroscopy, and the results are shown in Figures 3a. As expected, the three polymer samples showed characteristic absorption bands for push-pull conjugated polymers, with a strong absorption band centered at around
$700 \mathrm{~nm}$. Interestingly, the extension of the $\pi$-conjugation through the addition of thiophene units resulted in a slight hypsochromic shift of the principal absorption band, which was observed for both solution and thin-film analysis (Figures S15 and S16). As also observed in Figure S15, the relative intensities of the $0-0$ and $0-1$ absorption bands are changed upon $\pi$-conjugation extension, which can be directly associated with the formation of H-type aggregates. ${ }^{44,45}$ As previously observed for similar systems, both the hypsochromic shift of the absorption at $\lambda=700 \mathrm{~nm}$ and differences in the intensities of the $0-0$ and $0-1$ absorption bands with increasing thiophene chain length can be associated with an increased flexibility of the polymer backbone as thiophene units are added, resulting in a possible reduction in the effective conjugation length and reduced J-aggregation. ${ }^{46}$ More importantly, similar trends are observed for the conjugated polymer nanoparticles prepared using $\mathrm{HA}$ and Tween 80 (Figure 3a) as surfactants. For the nanoparticle solutions, the intensity of the $0-1$ absorption band was found to be systematically higher than the intensity of the $0-0$ absorption band, which also suggests the formation of $\mathrm{H}$-type aggregates in the SPNs. Despite the fact that no significant spectral shifting was observed before and after nanoprecipitation, the important increase in $0-1$ absorption band intensity after the nanoprecipitation process can be associated with a general reduction in $\mathrm{J}$-aggregation and the formation of $\mathrm{H}$-type aggregates as the nanoparticles are formed. Importantly, the UV-vis spectra show very little difference between nanoparticles formed from HA and Tween 80, which suggests that the surfactant does not play a strong role in the molecular organization of the DPP-based polymers during nanoparticle formation.

Further UV-vis experiments were performed to probe for the influence of the $\pi$-conjugation length on the aggregation of the polymers and nanoparticles in solution. The concept of $\mathrm{H}$ and J-type aggregation was designed to explain the spectroscopic observations in small molecules with excitons that are Frenkel-like. In conjugated polymers, the individual monomers are chemically tethered to each other, resulting in an inherent J-type interaction; ${ }^{47}$ furthermore, polymer chains can stack on top of each other, resulting in an H-type interaction. Therefore, when considering the photophysics of a 

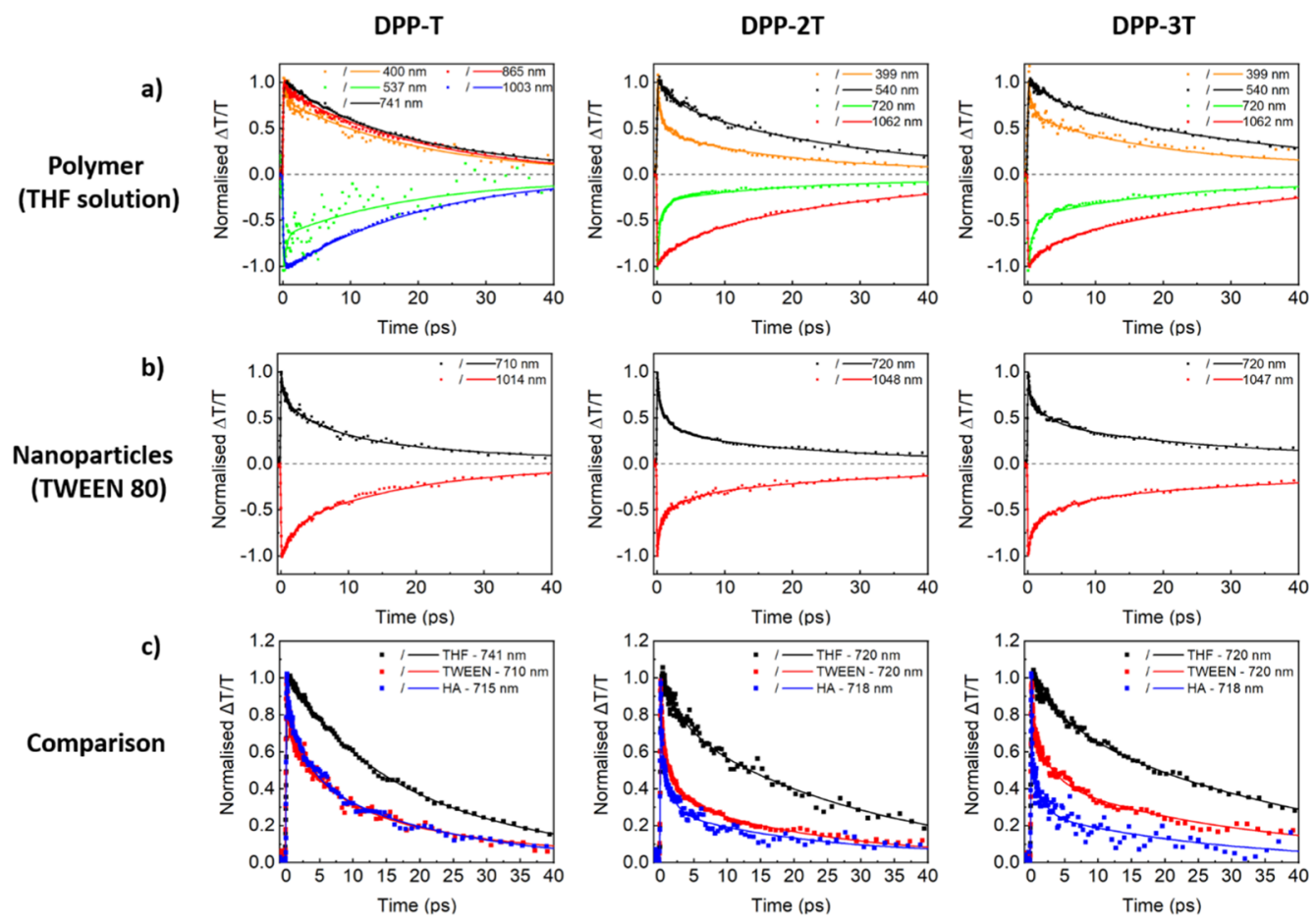

Figure 4. (a) Normalized decay traces from fs-TA measurements of (a) P(DPP-T) (left), P(DPP-2T) (middle), and P(DPP-3T) (right) in THF solution (select traced from the UV, VIS, and NIR windows are shown); (b) NP(DPP-T) (left), NP(DPP-2T) (middle), and NP(DPP-3T) (right) prepared from Tween 80 (select traced from the UV, VIS, and NIR windows are shown); and (c) normalized decay traces showing groundstate recovery of the polymers in THF solution and of the nanoparticles prepared from TWEEN and HA. All measurements made with a $795 \mathrm{~nm}$ excitation.

conjugated polymer, one must consider aggregates with a dual $\mathrm{HJ}$ nature. ${ }^{48}$ Consequently, an understanding of this dual $\mathrm{HJ}$ aggregation can lead to a further understanding of the bandwidth and coherence length of generated excitons. One way to determine whether a polymer aggregate is $\mathrm{H}$ - or $\mathrm{J}$ dominant is to examine the relative intensities of the $0-0$ and $0-1$ absorption bands. ${ }^{49,50}$ If the $0-0 / 0-1$ ratio $\left(A_{0-0} / A_{0-1}\right)$ is greater than 1 , the polymer $\mathrm{HJ}$ aggregates are dominated by intrachain $\mathrm{J}$ aggregation, where as if $A_{0-0} / A_{0-1}$ is less than 1 , the $\mathrm{HJ}$ polymer aggregate is dominated by interchain $\mathrm{H}$ aggregations. Therefore, in order to probe the aggregation of the investigated polymers in solution, the polymers were dissolved in chlorobenzene and heated to a temperature between 70 and $80{ }^{\circ} \mathrm{C}$. The absorption spectrum of the polymers was then recorded under controlled cooling of the samples, such that the UV-vis absorption was recoded every 5 ${ }^{\circ} \mathrm{C}$. The results of this experiment are summarized in Figure $3 \mathrm{~b}$ and Table S2. When the polymer solutions are heated, the absorption spectra for all polymers are blue-shifted and the intensity of the $0-1$ absorption band becomes higher than the 0-0 absorption band. As the solutions are then cooled, the spectra underwent a red shift and the relative intensities of the vibronic bands are reversed, resulting in a more intense $0-0$ absorption at low temperature. The relative peak intensities as a function of temperature are detailed in Figure S17. This observation confirms that the polymers are aggregated in solution and that $\mathrm{H}$-type (interchain) aggregation is favored as the temperature increases. As expected, as the solutions are cooled, the polymers rearrange again to form aggregates that favor J-type (intrachain) interactions. This phenomenon has been reported for other $\pi$-conjugated systems, such as poly(3hexylthiophene). ${ }^{43,51}$ Furthermore, no indication of polymer degradation was noted throughout the experiment, indicating that the polymers exhibit a good thermal stability in solution. A similar experiment was carried out on a solution of NP(DPP$\mathrm{T})$, where, interestingly, no spectral shift or change in relative band intensity was noted (see Figure S18). This again confirms that the SPN dispersions are stable at high temperature and furthermore shows that the polymer chains are tightly aggregated in the nanoparticle, making it such that they are not able to rearrange upon heating.

As previously observed for similar conjugated polymer systems, $\mathbf{P}(\mathbf{D P P}-\mathrm{T}), \mathbf{P}(\mathbf{D P P}-2 \mathrm{~T})$, and $\mathbf{P}(\mathbf{D P P}-3 \mathrm{~T})$ were found to be nonluminescent in THF solution. ${ }^{52}$ Therefore, because of this lack of emission, TA spectroscopy in THF was required to determine the excited-state lifetimes and kinetics of the polymers before and after nanoprecipitation. As detailed in the Supporting Information, the samples were excited with the fundamental output of the Ti-sapphire laser at $795 \mathrm{~nm}$ and were probed using a white light continuum generated by passing the $795 \mathrm{~nm}$ pulse through a sapphire window. Given the broad absorption range of the new materials, three separate spectral windows were evaluated when possible. The TA results are summarized in Figure 4 and are detailed in Figures S19-S22 for polymer solutions in THF and Figures S23-S28 for SPN solutions. Overall, the measured TA spectra for all polymers before nanoprecipitation are similar to those which have been previously reported for other $\pi$-conjugated DPPbased polymers. ${ }^{53}$ The UV spectral window $(350-590 \mathrm{~nm}$ ) revealed a ground-state bleach signal corresponding to the high energy absorption band (Figure 3 ) assigned to the aromatic thiophene units. A small photoinduced absorption signal can also be observed, which can be assigned to the $S_{0} \rightarrow S_{n}$ absorption of the thiophene units. The visible light (VIS) 
Table 2. Excited-State Lifetimes of Semiconducting Polymers in THF Solution and of SPN Solutions Obtained by TA Spectroscopy with a $795 \mathrm{~nm}_{\text {Excitation }}{ }^{a}$

\begin{tabular}{|c|c|c|c|c|}
\hline \multirow[b]{2}{*}{ polymer/nanoparticle } & \multirow{2}{*}{$\frac{\text { polymer solution }(\mathrm{THF})}{\text { VIS window }}$} & \multicolumn{2}{|c|}{ nanoparticle dispersion (polysorbate 80 ) } & \multirow{2}{*}{$\frac{\text { nanoparticle dispersion (HA) }}{\text { VIS window }}$} \\
\hline & & VIS window & NIR window & \\
\hline DPP-T & $\begin{array}{l}9.5 \pm 0.1 \mathrm{ps} \\
25.3 \pm 0.1 \mathrm{ps} \\
>8 \mathrm{~ns} \\
\mathrm{RMSE}=9.9 \times 10^{-5} \\
\mathrm{IRF}=115 \mathrm{fs}\end{array}$ & $\begin{array}{l}748 \pm 7 \mathrm{fs} \\
6.0 \pm 0.1 \mathrm{ps} \\
26.2 \pm 0.2 \mathrm{ps} \\
>8 \mathrm{~ns} \\
\mathrm{RMSE}=1.0 \times 10^{-4} \\
\mathrm{IRF}=72.1 \mathrm{fs}\end{array}$ & $\begin{array}{l}1.8 \pm 0.1 \mathrm{ps} \\
17.9 \pm 0.1 \mathrm{ps} \\
3.7 \pm 0.1 \mathrm{~ns} \\
\mathrm{RMSE}=1.4 \times 10^{-4} \\
\mathrm{IRF}=57.5 \mathrm{fs}\end{array}$ & $\begin{array}{l}89.9 \pm 2 \mathrm{fs} \\
2.65 \pm 0.1 \mathrm{ps} \\
20.1 \pm 0.1 \mathrm{ps} \\
6.6 \pm 0.2 \mathrm{~ns} \\
\mathrm{RMSE}=8.1 \times 10^{-5} \\
\mathrm{IRF}=52.8 \mathrm{fs}\end{array}$ \\
\hline DPP-2T & $\begin{array}{l}3.0 \pm 0.1 \mathrm{ps} \\
30.0 \pm 0.1 \mathrm{ps} \\
\mathrm{RMSE}=1.7 \times 10^{-4} \\
\mathrm{IRF}=95.2 \mathrm{fs}\end{array}$ & $\begin{array}{l}184.3 \pm 0.7 \mathrm{fs} \\
1.9 \pm 0.1 \mathrm{ps} \\
23.2 \pm 0.1 \mathrm{ps} \\
3.24 \pm 0.04 \mathrm{~ns} \\
\mathrm{RMSE}=7.2 \times 10^{-5} \\
\mathrm{IRF}=63.2 \mathrm{fs}\end{array}$ & $\begin{array}{l}398 \pm 2 \mathrm{fs} \\
3.1 \pm 0.1 \mathrm{ps} \\
27.5 \pm 0.1 \mathrm{ps} \\
4.38 \pm 0.03 \mathrm{~ns} \\
\mathrm{RMSE}=1.4 \times 10^{-4} \\
\mathrm{IRF}=59.6 \mathrm{fs}\end{array}$ & $\begin{array}{l}62.7 \pm 0.8 \mathrm{fs} \\
1.38 \pm 0.01 \mathrm{ps} \\
20.6 \pm 0.1 \mathrm{ps} \\
1.7 \pm 0.1 \mathrm{~ns} \\
\mathrm{RMSE}=7.8 \times 10^{-5} \\
\mathrm{IRF}=62.8 \mathrm{fs}\end{array}$ \\
\hline DPP-3T & $\begin{array}{l}4.5 \pm 0.1 \mathrm{ps} \\
38.7 \pm 0.1 \mathrm{ps} \\
6.8 \pm 0.6 \mathrm{~ns} \\
\mathrm{RMSE}=1.2 \times 10^{-4} \\
\mathrm{IRF}=105 \mathrm{fs}\end{array}$ & $\begin{array}{l}178 \pm 1 \mathrm{fs} \\
2.8 \pm 0.1 \mathrm{ps} \\
30.7 \pm 0.1 \mathrm{ps} \\
3.43 \pm 0.08 \mathrm{~ns} \\
\mathrm{RMSE}=6.5 \times 10^{-5} \\
\mathrm{IRF}=60.1 \mathrm{fs}\end{array}$ & $\begin{array}{l}533 \pm 3 \mathrm{fs} \\
4.5 \pm 0.1 \mathrm{ps} \\
37.9 \pm 0.1 \mathrm{ps} \\
4.58 \pm 0.04 \mathrm{~ns} \\
\mathrm{RMSE}=7.7 \times 10^{-5} \\
\mathrm{IRF}=55.7 \mathrm{fs}\end{array}$ & $\begin{array}{l}66.7 \pm 0.7 \mathrm{fs} \\
1.6 \pm 0.1 \mathrm{ps} \\
29.1 \pm 0.1 \mathrm{ps} \\
>8 \mathrm{~ns} \\
\mathrm{RMSE}=7.7 \times 10^{-5} \\
\mathrm{IRF}=56.0 \mathrm{fs}\end{array}$ \\
\hline
\end{tabular}

${ }^{a}$ Dominant lifetimes are shown in bold. RMSE = root-mean-squared error (describing the quality of the fit to the experimental data). IRF $=$ instrument response function (full width at half-maximum of the measured laser pulse).

window $(530-770 \mathrm{~nm})$ showed the ground-state bleach signal corresponding to the blue edge of the DPP absorption (Figure $3)$. The NIR window $(860-1100$ or $925-1100 \mathrm{~nm})$ showed the red edge of the ground-state bleach and a broad photoinduced absorption band corresponding to the $S_{0} \rightarrow S_{n}$ absorption of the excited state. As the ground-state absorption shifts to blue with an increasing amount of thiophene units in the $\pi$-conjugated backbone, the ground-state bleach is also shifted, making it more apparent in the VIS window and less apparent in the NIR window as expected from steady-state measurements.

When we examine the TA spectra in THF, we see that the ground-state bleach and the excited-state absorptions all relax with the same kinetics, suggesting that no intermediate excited states are formed between excitation and the time required to return to the ground state. To adequately fit the measured data, three to four exponential decay constants were applied. The very rapid ( $\mathrm{fs}$ ) relaxations are assigned to the relaxation of the vibronic states. The long-lifetime components are assigned a lifetime of several nanoseconds (note that the delay line has a limit of $8 \mathrm{~ns}$ ) and are assigned to a weak population of the triplet state. Finally, the dominant lifetime components are pslifetime components that are observed in all three spectral windows. These dominant lifetime components are assigned the excited-state lifetime of the polymers in THF solution at room temperature. Normalized decay traces for each of the polymers are provided in Figure $4 \mathrm{a}$, and the lifetime values are summarized in Tables 2 and S3 and S4. The TA maps as well as spectral slices and additional decay traces are provided in the Supporting Information.

When we examine the TA spectra of $\mathbf{P}($ DPP-T $)$, we are able to extract excited-state lifetimes of 20.4, 25.3, and 21.2 ps in the UV, VIS, and NIR windows, respectively (Table S3), leading to an average measured excited-state lifetime of 22.3 ps. Decay traces (Figures 4a and S22) showing the decay of the excited-state absorption intensity and the ground-state recovery all appear to have similar profiles and are clearly dominated by a $\sim 20$ ps lifetime component. The rapid loss of intensity in the first few picoseconds after photoexcitation, which can be seen in the 400 and $537 \mathrm{~nm}$ kinetic slices (Figure $\mathrm{S} 19$ ), is associated with the rapid vibronic relaxation that occurs on a femtosecond time scale. This rapid relaxation is less apparent in the 741, 865, and $1003 \mathrm{~nm}$ slices but is still present after deconvolution. The decay of the excited-state absorptions and the recovery of the ground-state bleach show nearly identical kinetics, and the absence of new transient signals or the presence of a rise time indicate that no intermediate excited-state species are formed after photoexcitation. The lack of these features and the short excitedstate lifetime further suggests a tightly bound electron-hole pair, which rapidly recombines, which is supported by the short conjugation length (approximately three units) that has been reported for a similar structure. ${ }^{52}$

After nanoprecipitation of $\mathbf{P}(\mathbf{D P P}-\mathrm{T})$ to form an aqueous dispersion of NP(DPP-T) that is stabilized by Tween 80 and $\mathrm{HA}$, the excited-state relaxation behavior was found to be relatively unchanged compared to that observed in THF solution. Because of the limitation in signal intensity, the probing of the UV window was found to be particularly challenging. However, a sufficient signal intensity for the measurement of the VIS and NIR windows was possible, which allowed for the observation of the blue edge of the DPP absorption (ground-state bleach) and the photoinduced absorption of the excited state, respectively. Once again, no intermediate excited states were found, which suggests that there is no charge separation or formation of new species in the excited stare of the nanoparticles. Select decay traces for the nanoparticle relaxation are shown in Figure $4 \mathrm{~b}$. The dominant lifetime for NP(DPP-T) prepared in Tween 80 was determined to be 26.2 and 17.9 ps in the VIS and NIR window, while the lifetime of the nanoparticles prepared in HA was measured to be 20.1 ps. Decay traces for the ground-state recovery of the nanoparticles prepared from Tween 80 and HA and the corresponding polymer in THF solution are shown in 
Figure 4c. Interestingly, NP(DPP-T) showed a recovery of the ground-state bleach at a faster overall rate than the native polymer in THF. Deconvolution shows, however, that this is due to an increased contribution of the rapid vibrational relaxation pathways, explained by the tight aggregation of the polymers in the nanoparticle which are able to knock against each other, leading to an increased contribution from the rapid vibrational relaxation pathways. Importantly, the relaxation kinetics of the nanoparticles formed from Tween 80 and HA are nearly identical, which once again suggests that the surfactant does not play an important role in the molecular organization and photophysical behavior of the nanoparticles. It is important to mention that, similar to the conjugated polymers before nanoprecipitation, nanoparticle solutions were found to be nonemissive, which can most likely be attributed to a quenching phenomenon caused by molecular aggregation.

A similar investigation was performed for P(DPP-2T), which shows that there is a larger contribution from the fast fsdecay that is associated with the vibrational relaxation of the excited states. This can likely be associated with an increased flexibility of the polymer chain. The decay of the photoinduced absorption signal in the NIR and the recovery of the groundstate bleach are largely dominated by lifetimes of 30.4 and 30.0 ps (average $30.2 \mathrm{ps}$ ), respectively. Interestingly, the UV window showed slightly different kinetics. In this window, the relaxation was found to be dominated by the 131 fs lifetime component, although there are also significant contributions of the 893 and 18.5 ps components (Figure S20 and Table S3). This very rapid relaxation is most likely due to the rapid vibrational relaxation of the oligothiophene linker. This rapid relaxation does not appear to be associated with any kind of rise in the photoinduced absorption signal of the DPP units, suggesting that the increased relaxation is not associated with energy transfer to the DPP centers. As observed for P(DPP$\mathrm{T})$, the nanoprecipitation of $\mathbf{P}(\mathrm{DPP}-2 \mathrm{~T})$ to generate NP(DPP-2T) did not significantly influence the shape of the TA spectrum. Analysis of the NP(DPP-2T) solutions yields excited-state lifetimes of 23.2 ps (VIS window) and 27.5 ps (NIR window) in Tween 80 and 20.6 ps in HA, which compares favorably with the lifetime of 30.0 ps that was obtained in THF solution (Table 1). Once again, Figure $4 \mathrm{~b}$ shows the ground-state recovery and the excited-state decay of the nanoparticle solution prepared in Tween 80, while Figure $4 \mathrm{c}$ shows a comparison of the excited-state relaxation between the polymer in THF solution and the nanoparticles. Examination of the TA results for P(DPP-3T) yields similar results. The average lifetime of the polymer in THF solution was determined to be 36.7 ps. After nanoprecipitation, an average lifetime of $34.3 \mathrm{ps}$ is obtained for nanoparticles prepared from Tween, and a lifetime of 29.1 ps was obtained when HA is used. Overall, as we increase the number of thiophene units between DPP units in the $\pi$-conjugated backbone, the number of possible conformations of the polymer is increased, as well as the number of vibronic modes that are available. It is important to note that, in TA measurements, the fast relaxation associated with the rapid vibronic relaxation of the thiophene bridge changes drastically as the length of conjugation increases. These results show that the photophysical behavior of the DPP-oligothiophene polymers is not greatly affected by the formation of nanoparticles nor is it affected by the surfactant used to prepare the nanoparticles, indicating that solution-based analysis of conjugated polymers can yield results that will help to predict the behavior of their corresponding nanoparticle solutions.

\section{CONCLUSIONS}

In conclusion, new SPNs were prepared from a diketopyrrolopyrrole-based conjugated polymer and HA or polysorbate 80 by using nanoprecipitation. Conjugated polymers with different numbers of thiophene moieties in the $\pi$-conjugated backbone were used in order to probe for the influence of $\pi$ conjugation length on the formation, size, and photophysical properties of the new semiconducting polymers. Based on the results obtained from DLS, SANS, and TEM, spherical nanoparticles with average diameters ranging from 100 to $200 \mathrm{~nm}$ were obtained, independently of the $\pi$-conjugation length or stabilizing polymer. UV-visible and fluorescence spectroscopy confirmed that all the new nanoparticle samples possess similar optical properties, with maximal absorption in the NIR. Moreover, TA spectroscopy was used to evaluate the excited-state dynamics before and after nanoprecipitation. Interestingly, all nanoparticles, independently of the stabilizing polymer used or of the $\pi$-conjugation length, showed very fast relaxation, and the relaxation kinetics of the nanoparticles formed from Tween 80 and HA were found to be nearly identical, which suggests that the surfactant does not play an important role in the molecular organization and photophysical behavior of the nanoparticles. Based on the results obtained, HA and polysorbate 80 are two efficient stabilizing agents for nanoprecipitation, leading to new nanoparticles with good optical properties. Moreover, the absence of a significant influence of the stabilizing polymer on the photophysical properties after nanoprecipitation confirms that the system is mostly depending on the properties of the parent conjugated polymer. With absorption in the NIR wavelengths, optimal photophysical properties, and good stability as dispersions, the new SPNs are promising candidates for various applications, including as bioimaging agents. Moreover, the in-depth characterization of the photophysical properties opens new opportunities for the design of novel SPNs with new properties.

\section{EXPERIMENTAL SECTION}

Materials. Chemical reagents were purchased from SigmaAldrich Co. Canada, Matrix Scientific, or Oakwood Products Inc., and were used as received. Solvents used for organic synthesis were obtained from Sigma-Aldrich and purified with a solvent purifier system (Swagelok, Solon, Ohio, USA). Tris(dibenzylideneacetone)dipalladium(0)-chloroform adduct $\left(\mathrm{Pd}_{2}(\mathrm{dba})_{3} \cdot \mathrm{CHCl}_{3}\right)$ was purchased from Sigma-Aldrich and recrystallized following a reported procedure. ${ }^{35-37}$ Tween 80 (polysorbate 80) was purchased from Sigma-Aldrich and used without further purification. HA sodium was purchased from LKT Laboratories and used without further purification. $\mathrm{D}_{2} \mathrm{O}$ (99.9\% purity) was purchased from Cambridge Isotopes.

Measurements and Characterization. DLS measurements were obtained using a Zetasizer Nano ZS utilizing a $\mathrm{He}-\mathrm{Ne}$ laser at a wavelength of $633 \mathrm{~nm}$ and a power of 100VA at $25{ }^{\circ} \mathrm{C}$. TEM images were taken on a Hitachi HT7700. Samples prepared for molecular weight analysis were analyzed using an HLC-8321GPC/HT (high-temperature) instrument. The samples were prepared using $1 \mathrm{mg} / \mathrm{mL}$ of polymer sample in trichlorobenzene and were allowed to stir at $80{ }^{\circ} \mathrm{C}$ for $12 \mathrm{~h}$ prior to injection. The sample analysis was performed at 180 
${ }^{\circ} \mathrm{C}$ with a flow rate of $1.0 \mathrm{~mL} / \mathrm{min}$ with injection quantities of $300 \mu \mathrm{L}$ per sample. The data were collected and integrated using EcoSEC 8321GPC/HT software suite.

SANS measurements were conducted on the EQSANS instrument at the Spallation Neutron Source of Oak Ridge National Laboratory (Oak Ridge, TN). Measurements were recorded at $37{ }^{\circ} \mathrm{C}$ using a single instrument configuration: a frame-skipping mode with instrument choppers running at 30 $\mathrm{Hz}$, a sample-to-detector distance of $3.5 \mathrm{~m}$, and a minimal wavelength of $2 \AA$. This configuration yielded a Q-range of $\sim 0.006$ to $0.4 \AA^{-1}$. Data were treated using Mantid software. ${ }^{5}$ In brief, data were calibrated to an absolute scale using a porasil standard. The data were also corrected for detector sensitivity, sample transmission, and background to yield the final $I(Q)$ scattering curves. SANS data were analyzed using the openly available SASView (http://www.sasview.org/) using the $P(r)$ inversion tool that calculates a real-space distance distribution function, $P(r)$, to determine the radius of gyration $\left(R_{\mathrm{g}}\right)$. The particle form factor was modeled using a sphere with uniform scattering length density. In the case of the polysorbate 80 surfactant, two sphere populations were required, and the surfactant micelle form factor was accounted for prior to determining $R_{\mathrm{g}}$. The absorption measurements were made on an Agilent Technologies 8453 Diode Array $\mathrm{UV}-$ vis spectrometer, with a spectral resolution of $1 \mathrm{~nm}$ in a 1 $\mathrm{cm}$ quartz cuvette. The fs transient spectra were acquired on a homemade system using a Soltice/OPA-800CF (Spectra Physics) Ti-sapphire laser. Excitation was carried out using the fundamental output of the laser $\left(\lambda_{\text {exc }}=795 \mathrm{~nm}\right.$, rep. rate $=$ $500 \mathrm{~Hz}$; and spot size $\approx 500 \mu \mathrm{m}$ ). The white light continuum (probe) is generated inside a sapphire window and a custommade dual CCD camera of $64 \times 1024$ pixels sensitive between 200 and $1100 \mathrm{~nm}$ (S7030, spectronic devices) is used for detection. The delay line permitted to probe up to $8 \mathrm{~ns}$ with an accuracy of $\sim 4 \mathrm{fs}$. The results were analyzed with the program Glotaran (http://glotaran.org) permitting to extract a sum of i $\mathrm{n} \mathrm{d}$ e $\mathrm{p}$ e $\mathrm{n} \mathrm{d}$ e $\mathrm{n} \mathrm{t}$

e $x$ p o n e n ti a $1 \mathrm{~s}$ $I(\lambda, t)=C_{1}(\lambda) \times \mathrm{e}^{\left(-t_{1} / \tau\right)}+C_{2}(\lambda) \times \mathrm{e}^{\left(-t_{2} / \tau\right)}+\ldots$ that fits the whole three-dimensional transient map. Compactional analysis of the different polymers was performed by DFT; all geometries were fully optimized by using the M06-2X functional in conjunction with a 6-311G basis set, and the scan calculations were performed using the opt $=$ ModRedundant with Gaussian 09 program package. ${ }^{6}$ Cyclic voltammetry measurements were carried out using a CHIode threeelectrode electrochemical setup utilizing a glassy carbon working electrode, a silver/silver chloride reference electrode, and a platinum wire counter electrode in an anhydrous acetonitrile solvent. NMR spectra were recorded on Bruker NMR spectrometers (DPX 300 and DPX 300 US operating at $300 \mathrm{MHz}\left({ }^{1} \mathrm{H}\right)$ and $\left.75 \mathrm{MHz}\left({ }^{13} \mathrm{C}\right)\right)$. UV-vis spectroscopy was performed on a Varian UV/visible Cary 50 spectrophotometer. Thermal gravimetric analysis was performed on a Mettler Toledo TGA SDTA 851e. Nitrogen (99.999\%) was used to purge the systems at a flow rate of $60 \mathrm{~mL} / \mathrm{min}$. All samples were run in aluminum crucibles. TGA samples were held at 25 ${ }^{\circ} \mathrm{C}$ for $30 \mathrm{~min}$ before heated to $550{ }^{\circ} \mathrm{C}$ at a rate of $5{ }^{\circ} \mathrm{C} / \mathrm{min}$. FTIR spectroscopy was performed on a Bruker ALPHA FTIR Spectrometer using a Platinum ATR sampling module.

Nanoparticle Preparation by Nanoprecipitation. Selected conjugated polymer $(1 \mathrm{mg})$ and $2 \mathrm{mg}$ of the selected surfactant were dissolved in $1 \mathrm{~mL}$ of THF and left to stir for 30 min. The solution was then injected in $9 \mathrm{~mL}$ of deionized water under probe sonication (amplitude of 60, power of $55 \mathrm{~W}$ for $2 \mathrm{~min}$ ). After sonication, the sample was passed through a $0.2 \mu \mathrm{m}$ PTFE (hydrophobic) syringe filter to remove any large aggregated materials. The nanoparticles were washed multiple times with deionized water and the dispersions were stored at $4{ }^{\circ} \mathrm{C}$ prior to use.

\section{ASSOCIATED CONTENT}

\section{Supporting Information}

The Supporting Information is available free of charge at https://pubs.acs.org/doi/10.1021/acsomega.9b03402.

Detailed experimental procedures and complete characterization of materials (PDF)

\section{AUTHOR INFORMATION}

\section{Corresponding Author}

*E-mail: srondeau@uwindsor.ca.

ORCID

Adam Langlois: 0000-0001-5999-0414

Drew Marquardt: 0000-0001-6848-2497

Simon Rondeau-Gagné: 0000-0003-0487-1092

\section{Author Contributions}

"A.L. and G.T.M. have equally contributed to this work. All authors contributed to the manuscript. All authors have given approval to the final version of the manuscript.

\section{Funding}

This work was supported by NSERC through a Discovery Grant (RGPIN-2017-06611 and RGPIN-2018-04841). S.R.-G. also acknowledges the Faculty of Science and the Department of Chemistry and Biochemistry at the University of Windsor for financial support. A.L. thanks NSERC for a postdoctoral fellowship (PDF-516635-2018).

\section{Notes}

The authors declare no competing financial interest.

\section{ACKNOWLEDGMENTS}

The authors also thank the Carmichael group at the University of Windsor for helping with material characterization and preparation. Part of this research was conducted at ORNL's Spallation Neutron Source, sponsored by the Scientific User Facilities Division, Office of Basic Energy Sciences, U.S. Department of Energy. This work benefited from the use of the SasView application, originally developed under NSF award DMR-0520547. SasView contains a code developed with funding from the European Union's Horizon 2020 research and innovation programme under the SINE2020 project, grant agreement no. 654000 .

\section{REFERENCES}

(1) Bässler, H.; Köhler, A. Charge Transport in Organic Semiconductors. Top. Curr. Chem. 2012, 312, 1-65.

(2) Guo, X.; Baumgarten, M.; Müllen, K. Designing $\pi$-Conjugated Polymers for Organic Electronics. Prog. Polym. Sci. 2013, 38, 18321908.

(3) Moliton, A.; Hiorns, R. C. Review of Electronic and Optical Properties of Semiconducting $\pi$-Conjugated Polymers: Applications in Optoelectronics. Polym. Int. 2004, 53, 1397-1412.

(4) Nelson, T. L.; Young, T. M.; Liu, J.; Mishra, S. P.; Belot, J. A.; Balliet, C. L.; Javier, A. E.; Kowalewski, T.; McCullough, R. D. Transistor Paint: High Mobilities in Small Bandgap Polymer 
Semiconductor Based on the Strong Acceptor, Diketopyrrolopyrrole and Strong Donor. Adv. Mater. 2010, 22, 4617-4621.

(5) Gsänger, M.; Bialas, D.; Huang, L.; Stolte, M.; Würthner, F. Organic Semiconductors Based on Dyes and Color Pigments. Adv. Mater. 2016, 28, 3615-3645.

(6) Onge, P. B. J. S.; Ocheje, M. U.; Selivanova, M.; RondeauGagné, S. Recent Advances in Mechanically Robust and Stretchable Bulk Heterojunction Polymer Solar Cells. Chem. Rec. 2019, 19, 1008.

(7) Nelson, J. Polymer: fullerene bulk heterojunction solar cells. Mater. Today 2011, 14, 462-470.

(8) Müller, C. D.; Falcou, A.; Reckefuss, N.; Rojahn, M.; Wiederhirn, V.; Rudati, P.; Frohne, H.; Nuyken, O.; Becker, H.; Meerholz, K. Multi-Colour Organic Light-Emitting Displays by Solution Processing. Nature 2003, 421, 829-833.

(9) Botiz, I.; Stingelin, N. Influence of Molecular Conformations and Microstructure on the Optoelectronic Properties of Conjugated Polymers. Materials 2014, 7, 2273-2300.

(10) Ocheje, M. U.; Charron, B. P.; Nyayachavadi, A.; RondeauGagné, S. Stretchable Electronics: Recent Progress in the Preparation of Stretchable and Self-Healing Semiconducting Conjugated Polymers. Flexible Printed Electron. 2017, 2, 043002.

(11) Rim, Y. S.; Bae, S.-H.; Chen, H.; De Marco, N.; Yang, Y. Recent Progress in Materials and Devices toward Printable and Flexible Sensors. Adv. Mater. 2016, 28, 4415-4440.

(12) O'connor, T. F.; Rajan, K. M.; Printz, A. D.; Lipomi, D. J. Toward Organic Electronics with Properties Inspired by Biological Tissue. J. Mater. Chem. B 2015, 3, 4947-4952.

(13) Torsi, L.; Magliulo, M.; Manoli, K.; Palazzo, G. Organic FieldEffect Transistor Sensors: A Tutorial Review. Chem. Soc. Rev. 2013, 42, 8612.

(14) Pu, K.; Chattopadhyay, N.; Rao, J. Recent Advances of Semiconducting Polymer Nanoparticles in in Vivo Molecular Imaging. J. Controlled Release 2016, 240, 312-322.

(15) Lyu, Y.; Pu, K. Recent Advances of Activatable Molecular Probes Based on Semiconducting Polymer Nanoparticles in Sensing and Imaging. Adv. Sci. 2017, 4, 1600481.

(16) Pu, K.; Shuhendler, A. J.; Jokerst, J. V.; Mei, J.; Gambhir, S. S.; Bao, Z.; Rao, J. Semiconducting Polymer Nanoparticles as Photoacoustic Molecular Imaging Probes in Living Mice. Nat. Nanotechnol. 2014, 9, 233-239.

(17) Qian, C.-G.; Zhu, S.; Feng, P.-J.; Chen, Y.-L.; Yu, J.-C.; Tang, X.; Liu, Y.; Shen, Q.-D. Conjugated Polymer Nanoparticles for Fluorescence Imaging and Sensing of Neurotransmitter Dopamine in Living Cells and the Brains of Zebrafish Larvae. ACS Appl. Mater. Interfaces 2015, 7, 18581-18589.

(18) Xu, X.; Liu, R.; Li, L. Nanoparticles Made of $\pi$-Conjugated Compounds Targeted for Chemical and Biological Applications. Chem. Commun. 2015, 51, 16733-16749.

(19) Liu, J.; Geng, J.; Liao, L.-D.; Thakor, N.; Gao, X.; Liu, B. Conjugated Polymer Nanoparticles for Photoacoustic Vascular Imaging. Polym. Chem. 2014, 5, 2854.

(20) Santra, S.; Dutta, D.; Walter, G. A.; Moudgil, B. M. Fluorescent Nanoparticle Probes for Cancer Imaging. Technol. Cancer Res. Treat. 2005, 4, 593-602.

(21) Yin, C.; Zhen, X.; Fan, Q.; Huang, W.; Pu, K. Degradable Semiconducting Oligomer Amphiphile for Ratiometric Photoacoustic Imaging of Hypochlorite. ACS Nano 2017, 11, 4174-4182.

(22) Sun, K.; Chen, H.; Wang, L.; Yin, S.; Wang, H.; Xu, G.; Chen, D.; Zhang, X.; Wu, C.; Qin, W. Size-Dependent Property and Cell Labeling of Semiconducting Polymer Dots. ACS Appl. Mater. Interfaces 2014, 6, 10802-10812.

(23) Cai, Y.; Liang, P.; Tang, Q.; Yang, X.; Si, W.; Huang, W.; Zhang, Q.; Dong, X. Diketopyrrolopyrrole-Triphenylamine Organic Nanoparticles as Multifunctional Reagents for Photoacoustic ImagingGuided Photodynamic/Photothermal Synergistic Tumor Therapy. ACS Nano 2017, 11, 1054-1063.

(24) Gao, Y.; Feng, G.; Jiang, T.; Goh, C.; Ng, L.; Liu, B.; Li, B.; Yang, L.; Hua, J.; Tian, H. Biocompatible Nanoparticles Based on Diketo-Pyrrolo-Pyrrole (DPP) with Aggregation-Induced Red/NIR
Emission for in Vivo Two-Photon Fluorescence Imaging. Adv. Funct. Mater. 2015, 25, 2857-2866.

(25) Zhen, X.; Feng, X.; Xie, C.; Zheng, Y.; Pu, K. Surface Engineering of Semiconducting Polymer Nanoparticles for Amplified Photoacoustic Imaging. Biomaterials 2017, 127, 97-106.

(26) Diao, H. J.; Wang, K.; Long, H. Y.; Wang, M.; Chew, S. Y. Highly Fluorescent and Photostable Polymeric Nanofibers as Scaffolds for Cell Interfacing and Long-Term Tracking. Adv. Healthcare Mater. 2016, 5, 529-533.

(27) Pu, K.; Mei, J.; Jokerst, J. V.; Hong, G.; Antaris, A. L.; Chattopadhyay, N.; Shuhendler, A. J.; Kurosawa, T.; Zhou, Y.; Gambhir, S. S.; et al. Diketopyrrolopyrrole-Based Semiconducting Polymer Nanoparticles for In Vivo Photoacoustic Imaging. Adv. Mater. 2015, 27, 5184-5190.

(28) Ranuncolo, S. M.; Ladeda, V.; Specterman, S.; Varela, M.; Lastiri, J.; Morandi, A.; Matos, E.; De Kier Joffe, E. B.; Puricelli, L.; Pallotta, M. A. G. Cd44 Expression in Human Gliomas. J. Surg. Oncol. 2002, 79, 30-36.

(29) Cai, Y.; Tang, Q.; Wu, X.; Si, W.; Zhang, Q.; Huang, W.; Dong, X. Bromo-Substituted Diketopyrrolopyrrole Derivative with Specific Targeting and High Efficiency for Photodynamic Therapy. ACS Appl. Mater. Interfaces 2016, 8, 10737-10742.

(30) Xu, Y.; Asghar, S.; Yang, L.; Chen, Z.; Li, H.; Shi, W.; Li, Y.; Shi, Q.; Ping, Q.; Xiao, Y. Nanoparticles Based on Chitosan Hydrochloride/Hyaluronic Acid/PEG Containing Curcumin: In Vitro Evaluation and Pharmacokinetics in Rats. Int. J. Biol. Macromol. 2017, 102, 1083-1091.

(31) Huang, Y.-W.; Wu, C.-S.; Chuang, C.-K.; Pang, S.-T.; Pan, T.M.; Yang, Y.-S.; Ko, F.-H. Real-Time and Label-Free Detection of the Prostate-Specific Antigen in Human Serum by a Polycrystalline Silicon Nanowire Field-Effect Transistor Biosensor. Anal. Chem. 2013, $85,7912-7918$.

(32) Calvo, P.; Gouritin, B.; Chacun, H.; Desmaële, D.; D’Angelo, J.; Noel, J. P.; Georgin, D.; Fattal, E.; Andreux, J. P.; Couvreur, P. LongCirculating Pegylated Polycyanoacrylate Nanoparticles as New Drug Carrier for Brain Delivery. Pharm. Res. 2001, 18, 1157-1166.

(33) Glaser, T.; Han, I.; Wu, L.; Zeng, X. Targeted Nanotechnology in Glioblastoma Multiforme. Front. Pharmacol. 2017, 8, 1-14.

(34) Wei, X.; Chen, X.; Ying, M.; Lu, W. Brain Tumor-Targeted Drug Delivery Strategies. Acta Pharm. Sin. B 2014, 4, 193-201.

(35) Zalesskiy, S. S.; Ananikov, V. P. $\mathrm{Pd}_{2}(\mathrm{dba})_{3}$ as a Precursor of Soluble Metal Complexes and Nanoparticles: Determination of Palladium Active Species for Catalysis and Synthesis. Organometallics 2012, 31, 2302-2309.

(36) Charron, B. P.; Ocheje, M. U.; Selivanova, M.; Hendsbee, A. D.; Li, Y.; Rondeau-Gagné, S. Electronic Properties of IsoindigoBased Conjugated Polymers Bearing Urea-Containing and Linear Alkyl Side Chains. J. Mater. Chem. C 2018, 6, 12070.

(37) Nyayachavadi, A.; Mason, G. T.; Nazir Tahir, M.; Ocheje, M. U.; Rondeau-Gagné, S. Covalent Crosslinking of Diketopyrrolopyrrole-Based Organogels with Polydiacetylenes. Langmuir 2018, 34, $12126-12136$

(38) Zhang, S.; Ocheje, M. U.; Huang, L.; Galuska, L.; Cao, Z.; Luo, S.; Cheng, Y. H.; Ehlenberg, D.; Goodman, R. B.; Zhou, D.; et al. The Critical Role of Electron-Donating Thiophene Groups on the Mechanical and Thermal Properties of Donor-Acceptor Semiconducting Polymers. Adv. Electron. Mater. 2019, 5, 1800899.

(39) Arnold, O.; Bilheux, J. C.; Borreguero, J. M.; Buts, A.; Campbell, S. I.; Chapon, L.; Doucet, M.; Draper, N.; Ferraz Leal, R.; Gigg, M. A.; et al. Mantid - Data Analysis and Visualization Package for Neutron Scattering and $\mu$ SR Experiments. Nucl. Instrum. Methods Phys. Res., Sect. A 2014, 764, 156-166.

(40) Moffitt, M.; Yu, Y.; Nguyen, D.; Graziano, V.; Schneider, D. K.; Eisenberg, A. Coronal Structure of Star-like Block Ionomer Micelles: An Investigation by Small-Angle Neutron Scattering. Macromolecules 1998, 31, 2190-2197.

(41) Vagberg, L. J. M.; Cogan, K. A.; Gast, A. P. Light-Scattering Study of Starlike Polymeric Micelles. Macromolecules 1991, 24, 16701677. 
(42) Glatter, O.; Kratky, O. Small Angle X-Ray Scattering; Academic Press: London, 1982.

(43) Cho, M. J.; Shin, J.; Hong, T. R.; Um, H. A.; Lee, T. W.; Kim, G. W.; Kwon, J. H.; Choi, D. H. Diketopyrrolopyrrole-Based Copolymers Bearing Highly $\pi$-Extended Donating Units and Their Thin-Film Transistors and Photovoltaic Cells. Polym. Chem. 2015, 6, 150-159.

(44) Printz, A. D.; Chiang, A. S.-C.; Savagatrup, S.; Lipomi, D. J. Fatigue in Organic Semiconductors: Spectroscopic Evolution of Microstructure Due to Cyclic Loading in Poly(3-Heptylthiophene). Synth. Met. 2016, 217, 144-151.

(45) Más-Montoya, M.; Janssen, R. A. J. The Effect of H- and JAggregation on the Photophysical and Photovoltaic Properties of Small Thiophene-Pyridine-DPP Molecules for Bulk-Heterojunction Solar Cells. Adv. Funct. Mater. 2017, 27, 1605779.

(46) Liu, F.; Wang, C.; Baral, J. K.; Zhang, L.; Watkins, J. J.; Briseno, A. L.; Russell, T. P. Relating Chemical Structure to Device Performance via Morphology Control in DiketopyrrolopyrroleBased Low Band Gap Polymers. J. Am. Chem. Soc. 2013, 135, 19248-19259.

(47) Spano, F. C. The Spectral Signatures of Frenkel Polarons in $\mathrm{H}$ and J Aggregates. Acc. Chem. Res. 2010, 43, 429-439.

(48) Spano, F. C.; Silva, C. H- and J-Aggregate Behavior in Polymeric Semiconductors. Annu. Rev. Phys. Chem. 2014, 65, 477500.

(49) Di Pietro, R.; Erdmann, T.; Carpenter, J. H.; Wang, N.; Shivhare, R. R.; Formanek, P.; Heintze, C.; Voit, B.; Neher, D.; Ade, H.; et al. Synthesis of High-Crystallinity DPP Polymers with Balanced Electron and Hole Mobility. Chem. Mater. 2017, 29, 10220-10232.

(50) Schroeder, B. C.; Chiu, Y.-C.; Gu, X.; Zhou, Y.; Xu, J.; Lopez, J.; Lu, C.; Toney, M. F.; Bao, Z. Non-Conjugated Flexible Linkers in Semiconducting Polymers: A Pathway to Improved Processability without Compromising Device Performance. Adv. Electron. Mater. 2016, 2, 1600104.

(51) Zhang, X.; Richter, L. J.; Delongchamp, D. M.; Kline, R. J.; Hammond, M. R.; McCulloch, I.; Heeney, M.; Ashraf, R. S.; Smith, J. N.; Anthopoulos, T. D.; et al. Molecular Packing of High-Mobility Diketo Pyrrolo-Pyrrole Polymer Semiconductors with Branched Alkyl Side Chains. J. Am. Chem. Soc. 2011, 133, 15073-15084.

(52) Mukhopadhyay, T.; Puttaraju, B.; Roy, P.; Dasgupta, J.; Meyer, A.; Rudnick, A.; Tscheuschner, S.; Kahle, F.-J.; Köhler, A.; Patil, S. Facile Synthesis and Chain-Length Dependence of the Optical and Structural Properties of Diketopyrrolopyrrole-Based Oligomers. Chem.Eur. J. 2017, 23, 13718-13723.

(53) Brady, M. A.; Ku, S.-Y.; Perez, L. A.; Cochran, J. E.; Schmidt, K.; Weiss, T. M.; Toney, M. F.; Ade, H.; Hexemer, A.; Wang, C.; et al. Role of Solution Structure in Self-Assembly of Conjugated Block Copolymer Thin Films. Macromolecules 2016, 49, 8187-8197. 